\title{
Modeling Hydrodynamic State of Oil and Gas Condensate Mixture in a Pipeline
}

\author{
Sergey Dudin ${ }^{1}$, Konstantin Voronin ${ }^{1, *}$, Svetlana Yakubovskaya $^{1}$ and Sharifjom \\ Mutavaliev $^{1}$ \\ ${ }^{1}$ Industrial University of Tyumen, 625001 Volodarskogo str. 38, Tyumen, Russia
}

\begin{abstract}
It is known that energy costs to pump a unit mass of hydrocarbon mixture in the form of gas $2 \div 3$ times exceed energy costs to pump hydrocarbon mixture in the form of liquid. As far as energy conservation during energy carrier transportation is concerned, an important task is development and application of a method to calculate gas-liquid hydrocarbons flow and heat and mass transfer in process and trunk pipelines during their design and operation.

The authors have developed a physical-mathematical model of a hydrocarbon flow in the gas condensate line based on balance equations of conservation of mass, impulse and energy of the transported medium within the framework of a quasi-1D approach.

Based on the developed model a calculation method was obtained which is used to analyze hydrodynamic state and composition of hydrocarbon mixture in each ith section of the pipeline when temperature-pressure and hydraulic conditions change.
\end{abstract}

\section{Introduction}

Transportation of hydrocarbons is one of the most energy-intensive industries. From the literature it is known that energy costs to pump a unit mass of hydrocarbon raw in the form of gas $2 \div 3$ times exceed energy costs to pump it in the form of liquid. [10] As far as energy conservation during energy carrier transportation is concerned, an important task is development and application of a method to calculate gas-liquid hydrocarbons flow and heat and mass transfer in process and trunk pipelines during their design and operation [21].

Gas condensate fields have a number of thermodynamic characteristics [26] that must be considered both in their field development and during transportation and processing of gas condensate. A complicated phase behaviour of the gas condensate system, as well as the dependence of the extracted raw materials on the phase state of the deposit other conditions being equal, is a key aspect. Therefore, when designing gas condensate lines the crucial task is to select the most appropriate methods of calculating thermophysical properties and phase equilibrium of the transported gas condensate [22-25].

\footnotetext{
Corresponding author: voronin tsogu@mail.ru
} 


\section{Research object}

Methods of calculating thermophysical properties (TPP) and phase equilibria are reviewed in quite a lot of scientific publications and reference books $[2,3,9,11,13,14,18,19$ etc.]. However, when it comes to such complex systems as oil, gas condensate, natural gas and their by-products, using even the known techniques is a difficult task. It should also be taken into account that large part of calculation methods is designed for individual hydrocarbons and mixtures of known composition, that is why using them to calculate TPP of complex mixtures of unknown composition is not always possible or can even results in additional, hard-to-estimate errors [2].

At the department of Transportation of Hydrocarbon Resources in Tyumen State Oil and Gas University (TSOGU) the authors have developed a physical-mathematical model of a hydrocarbon mixture (HCM) flow in the gas condensate line considering phase transitions and changes in the phase composition.

\section{Methods}

A detailed description of the model is presented in $[4,6,7,8]$ according to which the main model closing relations are the following.

1) The dependence of the gas condensate mixture density on pressure and temperature.

Given the gas condensate line upstream initial conditions (mass flow rate of component - Gi, true density of gas condensate - $\rho \mathrm{sm} 0$, temperature - T0, pressure - p0, mass fractions of component - Nmi) let us find the density of a group of hydrocarbons hexane+ at the inlet of the rated pipeline section by the formula:

$$
\rho_{C 6+}=\frac{N m_{n}}{\frac{1}{\rho s m_{0}}-\sum_{i=1}^{n-1} \frac{N m_{i}}{M_{i}} v_{i}}
$$

wher vi - molar volume of other components calculated for both gas and liquid by a single Peng-Robinson equation of state.

Having determined the density of a group of hydrocarbons hexane+ by (1) we find boiling temperature $\mathrm{TbC6}+$, molar weight $\mathrm{MC} 6+$, critical pressure and temperature $\mathrm{TcC} 6+$, pcC6 + and eccentricity factor $\omega \mathrm{C} 6+$ using techniques from $[2,17]$. Using these data, mass fractions zip of HCM components are calculated. Therefore, a group of hydrocarbons hexane + is included in the gas condensate mixture as a single pseudo-component.

2) The viscosity of the condensate mixture is calculated at temperature of $20^{\circ} \mathrm{C}$ and pressure of $1 \mathrm{~atm}$ by the formula:

$$
\mu s m_{0}=\frac{\sum_{i=1}^{N} z_{i} \sqrt{M_{i}} \mu_{i n d}}{\sum_{i=1}^{N} z_{i} \sqrt{M_{i}}}
$$

Recalculation of viscosity on thermobaric conditions in different sections of the pipeline is carried out according to the formula:

$$
\mu \text { sm }=10 \frac{\lg \left(\mu s m_{0}\right)+1.4503 \cdot 10^{-3} \cdot\left(\frac{p}{10}-0.101325\right) \cdot\left(5.2054 \cdot \mu s m_{0}^{0.278}+0.0239\right)-3}{}
$$


3) The isobaric heat capacity of the individual components in the mixture is calculated using a database from [11]. Isobaric heat capacity of a group of hydrocarbons hexane+ is determined using the Watson-Nelson formula. The heat capacity of the entire mixture was calculated according to the additivity rule:

$$
C p s m=\sum_{i=1}^{N} z_{i} \frac{C p_{i}}{M_{i}}
$$

4) The soil heat transfer is found by:

$$
\alpha_{s}= \begin{cases}\frac{2 \cdot \lambda_{s}}{D \cdot \ln \left(\frac{2 \cdot H}{D}+\sqrt{\left(\frac{2 \cdot H}{D}\right)^{2}-1}\right)}, & \text { if } \frac{H}{D}>3 \text { (Forchheimer - Vlasov) } \\ \frac{2 \cdot \lambda_{s}}{D \cdot \ln \left(4 \frac{H_{p r}}{D}+\frac{1}{N u_{s}}\right)}, & \text { if } \frac{H}{D}<3 \text { (Arson - Kutateladze) }\end{cases}
$$

where $H$ - the depth of the pipeline occurrence in the soil, $\mathrm{m} ; H_{\mathrm{pr}}$ is found by:

$$
H_{p r}=H+H_{e} ; \quad H_{e}=H_{S N} \frac{\lambda_{S O}}{\lambda_{S N}},
$$

and also $\lambda_{\text {sn }}$ and $\lambda_{\text {so }}-$ the thermal conductivity coefficients for snow and soil:

$$
\lambda_{S N}=0,465 \frac{W}{m \cdot \mathrm{K}}, \quad \lambda_{S O}=1,4 \frac{W}{m \cdot \mathrm{K}}
$$

$\mathrm{N} u_{\text {so }}-$ the Nusselt number for soil:

$$
N u_{s o}=\frac{\alpha_{a i r} \cdot D}{\lambda_{\text {so }}} \text {, }
$$

where $\alpha_{\text {air }}$ - the heat exchange coefficient for air:

$$
\alpha_{\text {air }}=11,6 \frac{W}{m^{2} \cdot \mathrm{K}} .
$$

5) The distribution coefficient or equilibrium constant of the $i^{\text {th }}$ component of the hydrocarbon mixture $\mathrm{Ki}$ is the relation of its molar fraction in a vapor phase yi to its molar fraction in a liquid phase xi.

Composition and quantitative ratio of the equilibrium vapor and liquid phases is found using equations of phase concentrations of the mixture components $[1,20]$. 


$$
\begin{aligned}
& x_{i}=\frac{z_{i}}{V\left(K_{i}-1\right)+1} \\
& y_{i}=\frac{z_{i} K_{i}}{V\left(K_{i}-1\right)+1}
\end{aligned}
$$

Equations (5) and (6) are called equations of phase concentrations of the mixture components. They allow us to determine the molar fractions of components in vapor and liquid phases of the mixture composition zi at given values of distribution coefficients $\mathrm{Ki}$ and molar fraction of the vapor phase $\mathrm{V}$.

6) By definition, the actual volumetric gas content is equal to:

$$
\alpha_{g}=\frac{V_{g}}{V_{s m}}=\frac{V_{g}}{V_{g}+V_{L}}=\frac{1}{1+\frac{V_{L}}{V_{g}}}
$$

If gas and liquid volumes are found as a product of the molar volume of gas and liquid $\left(v_{\mathrm{g}}, v_{\mathrm{L}}-\right.$ calculated by the equation of state given the known phase compositions) and the number of moles of the mixture in the vapor and liquid state, respectively $\left(N_{V}, N_{L}\right)$,

$$
\alpha_{g}=\frac{1}{1+\frac{v_{L}}{v_{g}} \frac{N_{L}}{N_{V}}}=\frac{1}{1+\frac{v_{L}}{v_{g}}\left(\frac{1}{V}-1\right)}
$$

7) The flow regime of the gas-liquid mixture is determined by one of the known techniques, for example, by the methods shown in $[12,15,16]$.

\section{Results and discussion}

Based on the developed model a calculation method was obtained which is used to analyze hydrodynamic state and composition of hydrocarbon mixture in each ith section of the pipeline when temperature-pressure and hydraulic conditions change. The developed technique was tested on the hydrocarbon mixture of de-ethanized condensate and oil transported from northern oil and gas condensate fields via the gas condensate line to the refinery. Results of a computational [9] and parametric study of thermophysical parameters of oil and gas condensate mixture in various product pipeline sections are discussed in $[5$, 8] and are shown in Figure 1, 2. 


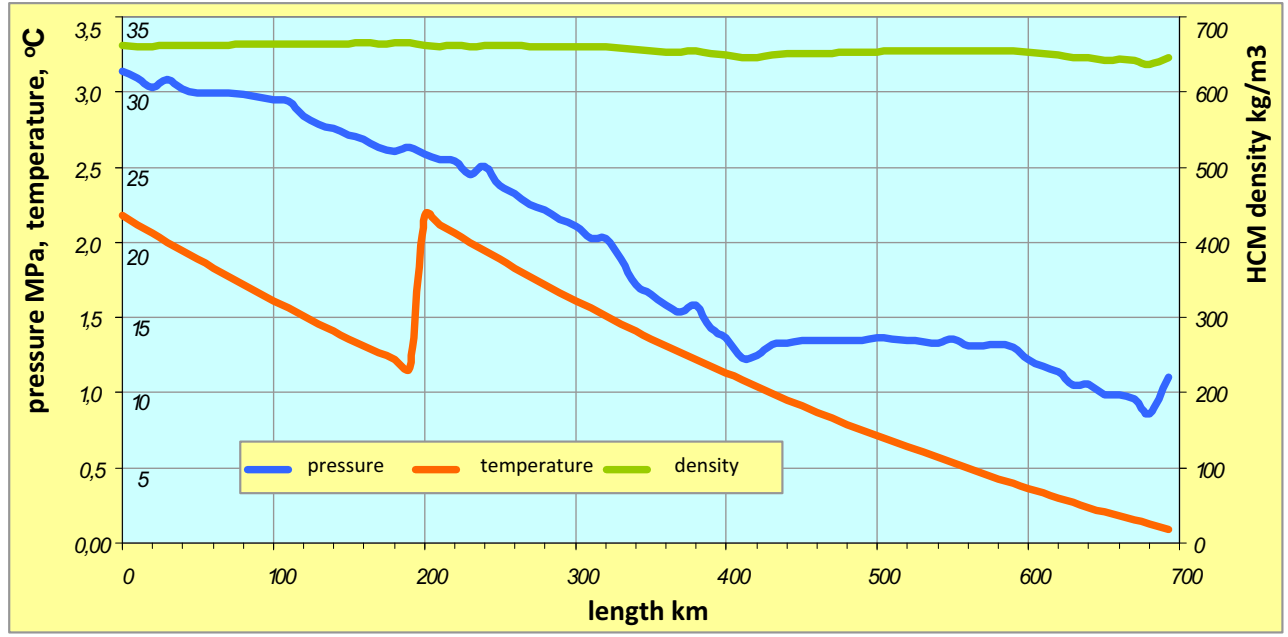

Fig. 1. HCM parameters along the gas condensate line.

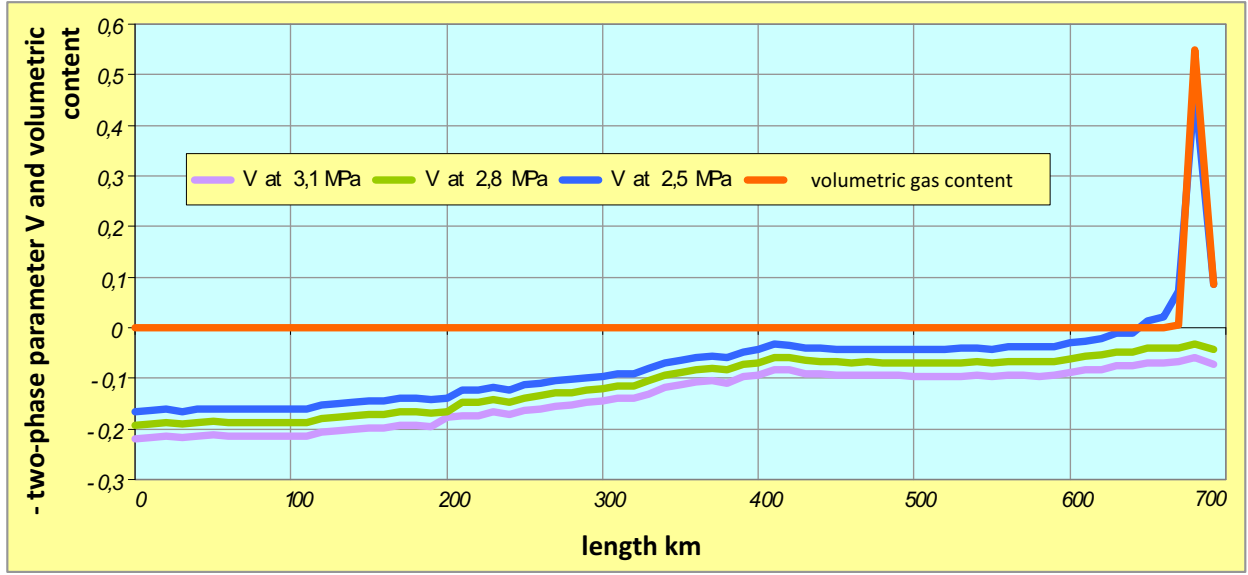

Fig. 2. Changes in HCM phase state parameters along the gas condensate line at differential product pressure in the inlet section.

Figure 1 shows the results of calculating HCM parameters along the gas condensate line. By comparing the calculated and actually observed differential pressure and temperature, we can conclude on the adequacy of the selected computational physical and mathematical model of the condensate line (relative deviation of the calculated differential pressure from actually observed is $4.6 \%$ and deviation of the estimated temperature change from the experimental was $1.5 \%$ ).

Figure 2 shows a computational and parametric study of HCM phase state along the gas condensate line, with the curves showing changes in the two-phase parameter $\mathrm{V}$ and the true volumetric gas content of hydrocarbons when lowering the operating pumping pressure by0.6 $\mathrm{MPa}$ at the beginning of the pipeline. It follows from these graphs that with a decrease in operating pumping pressure on the condensate line may arise areas where hydrocarbons enter the gas-liquid two-phase state (molar fraction of the vapor phase is 0 $<\mathrm{V}<1$ and acquires a definite physical meaning). In this case, the subsequent calculation of 
hydrodynamic parameters of the condensate line was carried out taking into account the two-phase flow of hydrocarbons.

\section{Conclusion}

1) On the basis of the developed physical and mathematical model of the gas-liquid media flow in a condensate line a technique and calculation algorithm were composed to determine the phases composition in an arbitrary section of the pipeline and to find the valid values of mass concentration of light hydrocarbons in the inlet condensate line section from the condition that a single-phase flow regime along the entire length of the pipeline is provided.

2) The developed technique can be used when managing unstable regimes of pumping fluids through pipelines, including the operational analysis of the hydrodynamic state of the condensate pipeline.

3) Results of a computational and parametric study show that in case of incomplete loading of the condensate line capacity there are areas with a two-phase flow. Therefore, in order to reduce energy consumption for transportation of condensate it is necessary to provide a single-phase flow regime along the entire length of the pipeline.

\section{References}

1. A. Brusilovsky, Phase transformations in the development of oil and gas (Graal, Moscow, 2002)

2. B. Grigoryev, A. Gerasimov, G. Lanchakov, Thermophysical properties and phase equilibria of gas condensates and their fractions (MPEI, Moscow, 2007)

3. Yu. Zemenkov, Diagnosis of damage and leakage during pipeline transport multiphase hydrocarbons (Vector Book, Tyumen, 2002)

4. S. Dudin, Yu. Zemenkov, A. Shabarov, N. Saranchyn, Oil and Gas, 6, 63-68 (2010)

5. S. Dudin, Yu. Zemenkov, A. Shabarov, N. Saranin, Pipeline transport: theory and practice, 5, 42-45 (2010)

6. S. Dudin, V. Nekrasov, Yu. Zemenkov, Oil and gas: a Separate edition of the Mining information and analytical Bulletin (research journal), 53-62 (2013)

7. S. Dudin, Yu. Zemenkov, Electronic journal "Oil and gas" (2007) Information on: http://www.ogbus.ru/authors/Dudin/Dudin_1.pdf.

8. S. Dudin, M. Zemenkova, Oil and gas terminal: Proceedings of international scientific-technical conference "Transportation and storage of hydrocarbons", 7, 8691 (2015)

9. S. Dudin, A. Shapovalov, Oil and gas terminal: Proceedings of international scientific-technical conference "Transportation and storage of hydrocarbons", $\mathbf{8}$ (2015)

10. A. Mirkin, V. Usins, Piping systems: reference edition (Chemistry, Moscow, 1971)

11. R. Reid, J. Prausnitz, T. Sherwood, Properties of gases and liquids: a reference guide (Chemistry, Leningrad, 1982)

12. A. Guzhov, V. Titov, V. Medvedev and others. Collection, transport and storage of natural hydrocarbon gases: a Training manual (Nedra, Moscow, 1978)

13. Yu. Zemenkov, Technical and parametric diagnostics in pipeline systems, (Vector Book, Tyumen, 2002)

14. Yu. Zemenkov, Monitoring of hydrodynamic and specifications piping systems: a tutorial (Vector Book, Tyumen, 2008) 
15. A. Shabarov, N. Saranin, V. Kutrunov, Proceedings of a Roundtable "Education through science and innovation", The modernization of education in conditions of globalization (2005)

16. V. Kurushina, Yu. Zemenkov, WIT Transactions on Ecology and the Environment, 190(2), 881-888 (2014)

17. D. Peng, D. Robinson, Ind. Eng. Chem. Fundam, 15, 59-64 (1976)

18. S. Stepanov, N., Cheremisin, S., Sokolov, A., Altunin, A., Shabarov, ECMOR 2012 13th European Conference on the Mathematics of Oil Recovery (2012) DOI: 10.399/2214-4609.20143270

19. R. Levitin, Yu. Zemenkov, Neftyanoe khozyaystvo - Oil Industry, 1, 110-114 (2016)

20. E. Kurushina, V. Kurushina, Life Science Journal, 11(11), 517-521 (2014)

21. R. Mamadaliev, V. Kuskov, Yu. Zemenkov, A. Popova, Applied Mechanics and Materials, 770, 19-22 (2015)

22. Yu. Zemenkov, V. Shalay, M. Zemenkova, Procedia Engineering, 113, 254-258 (2015)

23. Yu. Zemenkov, V. Shalay, M. Zemenkova, Procedia Engineering, 113, 312-315 (2015)

24. V. Antipyev, Yu. Zemenkov, N. Kudryavtseva, A method of calculation of losses of oil at accidents on the main oil pipelines (UMN Z and SZS, Tyumen, 1985)

25. G. Wilson, Modified Redlich-Kwong EOS Application to General Physical Data Calculations (Paper 15C presented at the 1968 AIChE Annual Meeting, Cleveland, Ohio, 1969) 